\title{
Coprime Irregular graphs
}

\section{S. Saravanakumar}

Abstract: An l-edge-weighting of a graph $G$ is a map $\emptyset: E(G) \rightarrow\{1,2,3, \ldots l\}$, where $l$ is a positive integer. For a vertex $v \in V(G)$, the weight $S_{\circledast}(v)$ is the sum of edge-weights appearing on the edges incident at $v$ under the edge-weighting $\emptyset$. An l-edge-weighting of $G$ is coprime irregular edge-weighting of $G$ if $\operatorname{gcd}\left(S_{\phi}(u), S_{\phi}(v)\right)=1$ for every pair of adjacent vertices $u$ and $v$ in G. A graph $G$ is coprime irregular if $G$ admits a coprime irregular edge-weighting. In this paper, we discuss this new irregular edge weighting of graphs.

Keywords: coprime, edge-weighting.

\section{INTRODUCTION}

All graphs considered in this article are simple, undirected and connected graphs. For graph theoretic terminology we refer to Chartrand and Lesniak [1].

Labeling of graphs is one of the fastest research areas which connects number theory and graph theory. Graph labeling was first introduced in the late 1960's and in recent years dozens of graphs labeling were introduced and studied by several authors. A graph labeling is an assignment of integers to the vertices or edges or both with respect to some conditions. A detailed survey of graph labeling is given in [2]. In this paper, we introduce a new variation of irregular labeling (irregular edge-weighting) of graphs namely coprime irregular edge-weighting of graphs.

\section{CLASSES OF COPRIME IRREGULAR GRAPHS}

In this section we prove that paths, cycles, triangular snakes, quadrilateral snakes and the corona of triangular and quadrilateral snakes are coprime irregular edge-weighting of a graph as follows. Now let us see the definition of coprime irregular edge-weighting of graphs as follows.

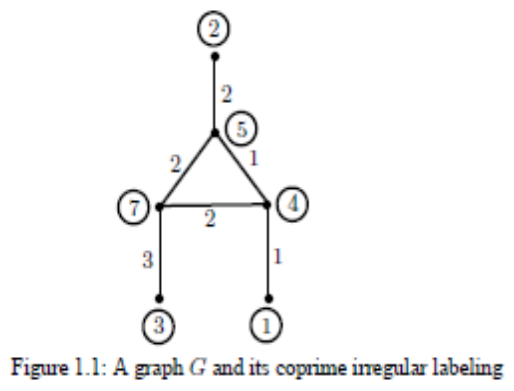

Definition 2.1. An l-edge-weighting of a graph $G=(V, E)$ is a $\operatorname{map} \emptyset: \mathrm{E}(\mathrm{G}) \rightarrow\{1,2,3, \ldots l\}$, where $l$ is a positive integer. For a vertex $v$ of $G$, the weight $S_{\phi}(v)$ is the sum of edge-weights appearing on the edges incident at $v$ under the edge-weighting $\emptyset$. An $l$-edge-weighting $\emptyset$ of $G$ is coprime

Revised Manuscript Received on December 16, 2019.

* Correspondence Author

S. Saravanakumar*, Department of Mathematics, Kalasalingam Academy of Research and Education, Krishnankoil, India.

Email: alg.ssk@gmail.com irregular if $\operatorname{gcd}\left(S_{\phi}(u), S_{\phi}(v)\right)=1$ for every pair of adjacent vertices $u$ and $v$ in $G$. A graph $G$ is said to be coprime irregular if $G$ admits a coprime irregular edge-weighting.

Example 2.2. A graph $G$ given in Figure 1.1 is coprime irregular.

Theorem 2.3. Path $P_{n}$ on $n \geq 3$ vertices is coprime irregular for all $n$.

Proof. Let $P_{n}=\left(v_{1}, v_{2}, v_{a}, \ldots v_{n}\right)$. We now prove this theorem by considering the following cases.

Case (i) $n$ is even.

Define an edge-weighting $\emptyset$ of $P_{\mathrm{n}}$ as follows. For all $1 \leq i \leq n-1$, let $\emptyset\left(v_{i} v_{i+1}\right)= \begin{cases}1 & \text { if } i \equiv 1 \text { or } 2(\bmod 4) \\ 2 & \text { otherwise }\end{cases}$

Then $S_{\phi}\left(\mathscr{V}_{1}\right)=1, S_{\phi}\left(\mathscr{V}_{n}\right)=1$ or 2 according as $n \equiv 2(\bmod 4)$ or $n \equiv 0(\bmod 4)$; and for each $2 \leq i \leq n-1$, we have

$$
S_{\emptyset}\left(v_{i}\right)=\emptyset\left(v_{i-1} v_{i}\right)+\emptyset\left(v_{i} v_{i+1}\right)= \begin{cases}4 & \text { if } i \equiv 0(\bmod 4) \\ 2 & \text { if } i \equiv 2(\bmod 4) \\ 3 & \text { otherwise. }\end{cases}
$$

It is not difficult to see that any two adjacent vertices of $P_{n}$ whose weights are coprime under $\emptyset$ and thus $P_{n}$ admits a coprime irregular edge-weighting.

Case (ii) $n$ is odd

Now, define for each $1 \leq i \leq n-1$,

$\emptyset\left(v_{i} v_{i+1}\right)= \begin{cases}1 & \text { if } i \equiv 2 \text { or } 3(\bmod 4) . \\ 2 & \text { otherwise }\end{cases}$

Then $S_{\phi}\left(v_{1}\right)=2, S_{\phi}\left(v_{n}\right)=1$ or 2 according as $n$ $\equiv 3(\bmod 4)$ or $n \equiv 1(\bmod 4)$; and for all $i=2,3, \ldots, n-1$, we have $S_{\varnothing}\left(v_{i}\right)=\emptyset\left(v_{i-1} v_{i}\right)+\emptyset\left(v_{i} v_{i+1}\right)$ 2 if $i \equiv 3(\bmod 4)$

$=\{$ if $i \equiv 0$ or $2(\bmod 4)$

4 otherwise.

Obviously, the weights of any two adjacent vertices of $P_{n}$ are coprime and thus $P_{n}$ is coprime irregular.

Theorem 2.4. For all $n \geq 4$, the cycle $C_{n}$ is coprime irregular. Proof. We prove this result in the following two cases.

Case (i) $n$ is odd

Considering the edge-weighting $\emptyset$ of $C_{\mathrm{n}}$ defined as follows.

For all $i=1,2,3, \ldots, n-1$, let

$\emptyset\left(v_{i} v_{i+1}\right)=\left\{\begin{array}{l}1 \text { if } i \equiv 1(\bmod 4) \\ 2 \text { if } i \equiv 2(\bmod 4) \\ 3 \text { if } i \equiv 3(\bmod 4) \\ 4 \text { if } i \equiv 0(\bmod 4)\end{array}\right.$

and let $\emptyset\left(v_{n} v_{1}\right)=1$ or 3 according as $n \equiv 1(\bmod 4)$ or $n \equiv 3$ $(\bmod 4)$. Then for any two adjacent vertices of $C_{n}$, their weights are coprime. Thus $\emptyset$ is coprime irregular edge-weighting of $C_{\mathrm{n}}$ and hence $C_{\mathrm{n}}$ is coprime irregular.

Case (ii) $n$ is even

Assign $\emptyset\left(v_{1} v_{2}\right)=1, \emptyset\left(v_{2} v_{a}\right)=2, \emptyset\left(v_{a} v_{4}\right)=3$ and $\emptyset\left(v_{4} v_{5}\right)$ $=4$ and for all the remaining vertices,

Published By:

Blue Eyes Intelligence Engineering 


\section{Coprime Irregular graphs}

$\emptyset\left(v_{i} v_{i+1}\right)=\left\{\begin{array}{l}1 \text { if } i \equiv 0(\bmod 4) \\ 2 \text { if } i \equiv 1(\bmod 4) \\ 3 \text { if } i \equiv 2(\bmod 4) \\ 4 \text { if } i \equiv 3(\bmod 4)\end{array}\right.$

One can easily verify that the weights of any two adjacent vertices of $C_{n}$ are coprime and so $\emptyset$ is a coprime irregular edge-weighting of $C_{\mathrm{n}}$. Hence $C_{\mathrm{n}}$ is coprime irregular.

Definition 2.5. A triangular snake $T S_{n}$ is obtained from a path $\left(u_{1}, u_{2}, \ldots, u_{n-1}, u_{n}\right)$ by joining $u_{i}$ and $u_{i+1}$ to a new vertex $\nu_{i}$ for $i=1,2, \ldots, n$. A quadrilateral snake $Q S_{n}$ is obtained from the path $\left(u_{1}, u_{2}, \ldots, u_{n-1}, u_{n}\right)$ by introducing $n$ copies of $K_{2}$, say $v_{1} w_{1}, v_{2} w_{2}, \ldots, v_{n} w_{n}$ and joining $v_{i}$ to $u_{i}$ and $w_{i}$ to $u_{i+1}$ for $i=1,2, \ldots, n$.

Theorem 2.6. The triangular snake graph $T S_{n}$ is coprime irregular for all $n$.

Proof. Suppose $n$ is even. Now we define an edge-weighting $\emptyset$ as follows. For all $1 \leq i \leq n$, let $\emptyset\left(u_{i} v_{i}\right)=1, \emptyset\left(v_{i} u_{i+1}\right)=4$ and $\emptyset\left(u_{i} u_{i+1}\right)=\left\{\begin{array}{ll}2 & \text { if } i \equiv 0 \text { or } 1(\bmod 4) \\ 4 & \text { otherwise }\end{array}\right.$.

For $n=8$, the graph $T S_{\mathrm{g}}$ and edge-weighting $\emptyset$ are shown in Figure 2.

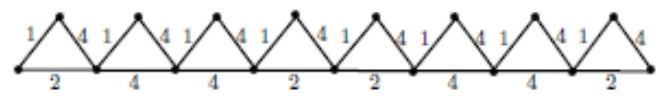

Figure 2

Certainly, by the definition of $\emptyset$, we have $S_{\emptyset}\left(u_{1}\right)=3$, $S_{\phi}\left(u_{n+1}\right)=6$ (or) 8 according as $n \equiv 0$ (mod 4) and $n \equiv 2(\bmod 4)$; and for all $i=2,3,4, \ldots, n$, we have

$$
\begin{aligned}
& S_{\varnothing}\left(u_{i}\right)=\emptyset\left(u_{i} v_{i-1}\right)+\emptyset\left(u_{i} v_{i}\right)+\emptyset\left(u_{i} u_{i+1}\right)+\emptyset\left(u_{i} u_{i-1}\right) \\
& = \begin{cases}9 & \text { if } i \equiv 1(\bmod 4) \\
11 & \text { if } i \equiv 0 \operatorname{or} 2(\bmod 4) . \\
13 & \text { if } i \equiv 3(\bmod 4)\end{cases}
\end{aligned}
$$

Clearly, the weights of any two adjacent vertices of $T S_{\mathrm{n}}$ are coprime and hence $\emptyset$ is a coprime irregular edge-weighting of $T S_{\mathrm{n}}$.

On the other hand, let we assume that $n$ is odd. Consider the edge-weighting $\emptyset$ of $T S_{n}$ defined as follows.

For all $i=1,2, \ldots, n, \emptyset\left(u_{i} v_{i}\right)=\varnothing\left(v_{i} u_{i+1}\right)=1$ and $\emptyset\left(u_{i} u_{i+1}\right)$

$$
=\left\{\begin{array}{l}
1 \text { if } i \equiv 2(\bmod 4) \\
2 \text { if } i \equiv 1(\bmod 4) \\
3 \text { if } i \equiv 0(\bmod 4) \\
4 \text { otherwise. }
\end{array}\right.
$$

For $n=9$, the graph $T S_{9}$ and its edge-weighting given in the following Figure 3.

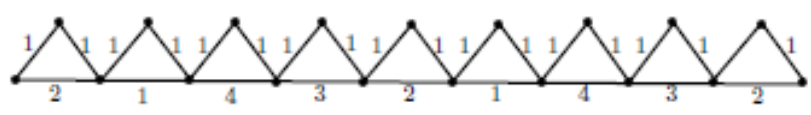

Figure 3

Then $S_{\phi}\left(u_{1}\right)=3, S_{\phi}\left(u_{n+1}\right)=3$ (or) 5 according as $n \equiv 1(\bmod 4)$ or $n \equiv 3(\bmod 4)$; and for all $i=1,2, \ldots, n$, we have

$$
\begin{aligned}
& S_{\varnothing}\left(u_{i}\right)=\emptyset\left(u_{i} v_{i-1}\right)+\emptyset\left(u_{i} v_{i}\right)+\emptyset\left(u_{i} u_{i+1}\right)+\emptyset\left(u_{i} u_{i-1}\right) \\
& = \begin{cases}5 & \text { if } i \equiv 2(\bmod 4) \\
9 & \text { if } i \equiv 0(\bmod 4) \\
7 & \text { otherwise. }\end{cases}
\end{aligned}
$$

Obviously, the weights of any two adjacent vertices of $T S_{n}$ is a coprime irregular.

Theorem 2.7. For all $n$, the quadrilateral snake graph $Q S_{n}$ is coprime irregular.

Proof. Consider the following cases.

Case 1. $n$ is even.

Consider the edge-weighting $\emptyset$ of quadrilateral snake defined as follows. For all $i=1,2, \ldots, n$,

$\emptyset\left(u_{i} v_{i}\right)=\emptyset\left(v_{i} w_{i}\right)=2, \emptyset\left(w_{i} u_{i+1}\right)=3$ and $\emptyset\left(u_{i} u_{i-1}\right)$

$= \begin{cases}1 & \text { if } i \equiv 2(\bmod 4) \\ 3 & \text { if } i \equiv 0 \text { or } 1(\bmod 4) \\ 5 & \text { otherwise. }\end{cases}$

For $n=6$, the graph $Q S_{6}$ and its edge-weighting given in Figure 4.

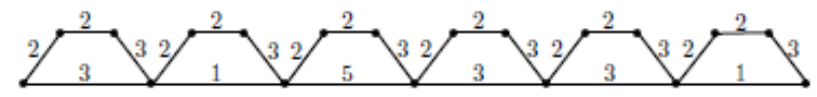

Figure 4

Then $S_{\phi}\left(u_{1}\right)=5, S_{\phi}\left(u_{n+1}\right)=4$ or 6 according as $n \equiv 3(\bmod 4)$ or $n \equiv 1(\bmod 4)$ and for all $i=1,2, \ldots, n$, we have $S_{\varnothing}\left(u_{i}\right)=\emptyset\left(u_{i} w_{i-1}\right)+\emptyset\left(u_{i} v_{i}\right)+\emptyset\left(u_{i} u_{i+1}\right)+\emptyset\left(u_{i} u_{i-1}\right)$ 9 if $i \equiv 2(\bmod 4)$

$=11$ if $i \equiv 1$ or $3(\bmod 4)$

13 otherwise.

Clearly, the weights of any two adjacent vertices are coprime and so $\emptyset$ is a coprime irregular edge-weighting of $Q S_{\mathrm{n}}$. Therefore the graph $Q S_{\mathrm{n}}$ is coprime irregular.

Case 2. $n$ is odd

Consider the edge-weighting $\emptyset$ of the quadrilateral snake defined as follows.

For all $i=1,2, \ldots, n$,

$\emptyset\left(u_{i} v_{i}\right)=\emptyset\left(v_{i} w_{i}\right)=3, \emptyset\left(u_{i+1} w_{i}\right)=4$ and $\emptyset\left(u_{i} u_{i-1}\right)=$ $\left\{\begin{array}{l}1 \text { if } i \equiv 2(\bmod 4) \\ 2 \text { if } i \equiv 1(\bmod 4) \\ 3 \text { if } i \equiv 0(\bmod 4) \\ 4 \text { if } i \equiv 3(\bmod 4)\end{array}\right.$

For $n=5$, the graph $Q S_{5}$ and its edge-weighting illustrated in Figure 5.

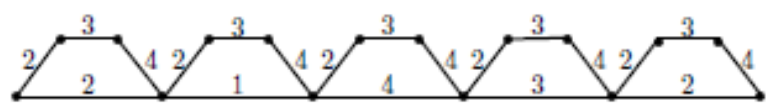

Figure 5 
Then $S_{\phi}\left(u_{1}\right)=4, S_{\phi}\left(u_{n+1}\right)=6$ or 8 according as $n \equiv 2(\bmod 4)$ or $n \equiv 0(\bmod 4)$ and for all $i=2,3, \ldots, n$, we have

$S_{\emptyset}\left(u_{i}\right)=\emptyset\left(u_{i} w_{i-1}\right)+\emptyset\left(u_{i} v_{i}\right)+\emptyset\left(u_{i} u_{i+1}\right)+\emptyset\left(u_{i} u_{i-1}\right)$

$S_{\varnothing}\left(u_{i}\right)= \begin{cases}9 & \text { if } i \equiv 2(\bmod 4) \\ 11 & \text { if } i \equiv 1 \text { or } 3(\bmod 4) \\ 13 & \text { otherwise. }\end{cases}$

Certainly from the above, the weights of any two adjacent vertices are coprime and so $\varnothing$ is a coprime irregular edge-weighting of $Q S_{\mathrm{n}}$. Hence $Q S_{n}$ is coprime irregular.

Definition 2.8. The corona $G^{+}$of a graph $G$ is the graph obtained from $G$ by attaching exactly one pendant edge at each of the vertices of $G$.

Theorem 2.9. Corona of a triangular snake graph is coprime irregular.

Proof. Consider the following cases.

Case 1. $n$ is even.

Define an edge-weighting $\emptyset$ of the corona of a triangular snake as follows.

For all $i=1,2,3, \ldots, n$, define

$\emptyset\left(u_{i} v_{i}\right)=\emptyset\left(u_{i+1} v_{i}\right)=\emptyset\left(v_{i} v_{i}^{f}\right)=\emptyset\left(u_{i} u_{i}^{f}\right)=1, \emptyset\left(u_{i} u_{i+1}\right)=$ $(2$ if $i \equiv 0(\bmod 4)$

3 if $i \equiv 1(\bmod 4)$

5 if $i \equiv 2(\bmod 4)$

6 if $i \equiv 3(\bmod 4)$

and for all $I=1,2,3,4, \ldots, n-1, \emptyset\left(u_{i} v_{i}\right)=\emptyset\left(u_{i+1} v_{i}\right)=\emptyset\left(v_{i} v_{i}^{f}\right)$ $=\emptyset\left(u_{i} u_{i}^{\prime}\right)=1$.

For the corona of a triangular snake $\mathrm{TS}_{6}$ as given in Figure 6.

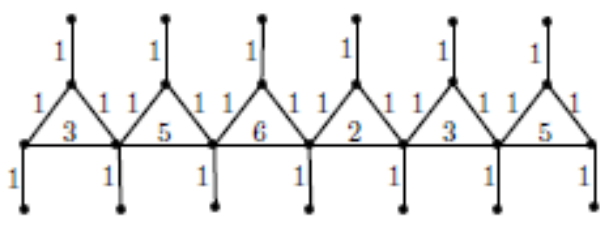

Figure 6

Then $S_{\phi}\left(v_{i}\right)=3, S_{\phi}\left(v_{i}^{f}\right)=1, S_{\phi}\left(u_{i}^{f}\right)=1$, for all $i=1,2,3$, $4, \ldots, n-1, \quad S_{\phi}\left(u_{1}\right)=5, S_{\phi}\left(u_{n}\right)=4$ or 7 according as $n \equiv 1(\bmod 4)$ or $n \equiv 3(\bmod 4)$ where $k \geq 0$ and for all $=$ $2,3,4, \ldots, n-1$, we have

$S_{\phi}\left(u_{i}\right)=\emptyset\left(u_{i} v_{i-1}\right)+\emptyset\left(u_{i} v_{i}\right)+\emptyset\left(u_{i} u_{i+1}\right)+\emptyset\left(u_{i} u_{i}^{i}\right)$

$S_{\phi}\left(u_{i}\right)=\left\{\begin{array}{l}8 \text { if } i \equiv 1(\bmod 4) \\ 11 \text { if } i \equiv 0 \text { or } 2(\bmod 4) \\ 14 \text { otherwise. }\end{array}\right.$

Clearly, the weights of any two adjacent vertices are relatively prime and hence $\emptyset$ is a coprime irregular edge-weighting of corona of a triangular snake. So that the corona of a triangular snake is coprime irregular edge-weighting and hence Case 1 follows.

Case 2. $n$ is odd

Define an edge-weighting $\emptyset$ of the corona of a triangular snake as follows. For all $i=1,2,3, \ldots, n-1$,

$\emptyset\left(u_{i} v_{i}\right)=\emptyset\left(u_{i+1} v_{i}\right)=3$ and $\emptyset\left(v_{i} v_{i}^{f}\right)=\emptyset\left(u_{i} u_{i}^{f}\right)=1$. $\emptyset\left(u_{i} u_{i+1}\right)= \begin{cases}2 & \text { if } i \equiv 1 \text { or } 2(\bmod 4) \\ 4 & \text { otherwise. }\end{cases}$

and for all $i=1,2,3, \ldots, n-1, \emptyset\left(u_{i} v_{i}\right)=\emptyset\left(u_{i+1} v_{i}\right)=3$ and $\emptyset\left(v_{i} v_{i}^{f}\right)=\emptyset\left(u_{i} u_{i}^{t}\right)=1$.
For the corona of a triangular snake $\mathrm{TS}_{5}$ as given in Figure 7.

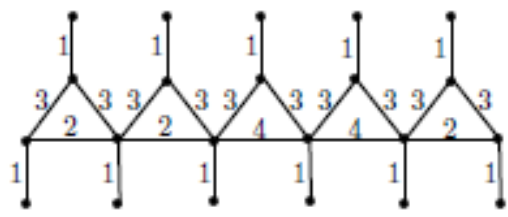

Figure 7

Then $S_{\phi}\left(\mathcal{V}_{\mathrm{i}}\right)=7, S_{\mathrm{\phi}}\left(\mathscr{V}_{\mathrm{i}}^{f}\right)=1, S_{\phi}\left(u_{\mathrm{i}}^{t}\right)=1$, for all $i=1,2,3$, $4, \ldots, n-1, \quad S_{\phi}\left(u_{1}\right)=6, S_{\phi}\left(u_{n}\right)=6$ or 8 according as $n \equiv 0(\bmod 4)$ or $n \equiv 2(\bmod 4)$ where $k \geq 0$ is an integer and for all $=2,3,4, \ldots, n-1$, we have

$S_{\varnothing}\left(u_{i}\right)=\emptyset\left(u_{i} v_{i-1}\right)+\emptyset\left(u_{i} v_{i}\right)+\emptyset\left(u_{i} u_{i+1}\right)+\emptyset\left(u_{i} u_{i}^{r}\right)+$ $\emptyset\left(u_{i} u_{i-1}\right)$

$S_{\phi}\left(u_{i}\right)=\left\{\begin{array}{l}11 \text { if } i \equiv 2(\bmod 4) \\ 13 \text { if } i \equiv 1 \operatorname{or} 3(\bmod 4) \\ 15 \text { otherwise. }\end{array}\right.$

Clearly, the weights of any two adjacent vertices are relatively prime and hence $\emptyset$ is a coprime irregular edge-weighting of corona of a triangular snake. So that the corona of a triangular snake is coprime irregular edge-weighting. Hence case 2.

Theorem 2.7. Corona of a quadrilateral snake graph is coprime irregular for all $n$.

Proof. We prove the theorem in the following cases.

Case 1. $n$ is odd

Define an edge-weighting $\emptyset$ of the corona of a quadrilateral snake as follows.

For all $i=1,2,3, \ldots, n-1$, assign

$\emptyset\left(u_{i} u_{i}^{f}\right)=\emptyset\left(v_{i} w_{i}^{\prime}\right)=\emptyset\left(v_{i} v_{i}^{f}\right)=1, \emptyset\left(u_{i} v_{i}\right)=2$ and $\emptyset\left(u_{i+1} v_{i}^{f}\right)=3$

$\emptyset\left(u_{i} u_{i+1}\right)=\left\{\begin{array}{l}2 \text { if } i \equiv 1(\bmod 6) \\ 3 \text { if } i \equiv 2(\bmod 6) \\ 4 \text { if } i \equiv 3(\bmod 6) \\ 5 \text { if } i \equiv 0(\bmod 6) \\ 6 \text { if } i \equiv 5(\bmod 6) \\ 7 \text { if } i \equiv 4(\bmod 6)\end{array}\right.$

For all $i=1,2,3, \ldots, n-1, \emptyset\left(u_{i} u_{i}^{t}\right)=\emptyset\left(v_{i} w_{i}^{t}\right)=\emptyset\left(v_{i} v_{i}^{f}\right)=1$, $\emptyset\left(u_{i} v_{i}\right)=2$ and $\emptyset\left(u_{i+1} v_{i}^{\prime}\right)=3$.

Then $S_{\phi}\left(v_{\mathrm{i}}\right)=4, S_{\phi}\left(v_{\mathrm{i}}^{f}\right)=5, S_{\phi}\left(w_{\mathrm{i}}\right)=S_{\phi}\left(w_{\mathrm{i}}^{f}\right)=S_{\phi}\left(u_{\mathrm{i}}^{f}\right)=1$, for all $i=1,2,3,4, \ldots, n-1, S_{\phi}\left(u_{1}\right)=5, S_{\phi}\left(u_{n}\right)=7$ (or) 9 (or) 11 according as $n \equiv 3(\bmod 6)$ or $n \equiv 1(\bmod 6)$ or $n \equiv 5(\bmod 6)$.

For all $i=2,3,4, \ldots, n-1$, we have

$S_{\varnothing}\left(u_{i}\right)=\emptyset\left(u_{i} v_{i-1}^{\prime}\right)+\emptyset\left(u_{i} v_{i}\right)+\emptyset\left(u_{i} u_{i+1}\right)+\emptyset\left(u_{i} u_{i}^{f}\right)+$ $\emptyset\left(u_{i} u_{i-1}\right)$

$S_{\phi}\left(u_{i}\right)=\left\{\begin{array}{l}11 \text { if } i \equiv 2(\bmod 6) \\ 13 \text { if } i \equiv 1 \text { or } 3(\bmod 6) \\ 17 \text { if } i \equiv 0 \text { or } 4(\bmod 6) \\ 19 \text { otherwise. }\end{array}\right.$

Clearly, the weights of any two adjacent vertices are coprime and hence $\emptyset$ is a coprime irregular edge-weighting of corona of a quadrilateral snake. So that the corona of a quadrilateral snake is coprime irregular edge-weighting.

Published By:

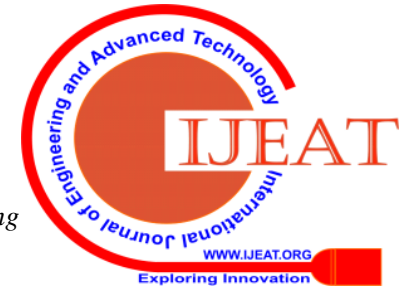




\section{Coprime Irregular graphs}

Case 2. $n$ is even

Define an edge-weighting $\emptyset$ of the corona of a quadrilateral snake as follows. For all $i=1,2, \ldots, n-1$,

$\emptyset\left(v_{i} w_{i}\right)=\emptyset\left(v_{i}^{f} w_{i}^{f}\right)=\emptyset\left(u_{i} t_{i}\right)=\emptyset\left(u_{i} t_{i}^{f}\right)=\emptyset\left(v_{i} v_{i}^{f}\right)=1$, $\emptyset\left(u_{i} v_{i}\right)=2$ and $\emptyset\left(u_{i+1} v_{i}^{\prime \prime}\right)=3$ and

$\emptyset\left(u_{i} u_{i+1}\right)=\left\{\begin{array}{l}1 \text { if } i \equiv 2(\bmod 4) \\ 2 \text { if } i \equiv 1(\bmod 4) \\ 3 \text { if } i \equiv 0(\bmod 4) \\ 4 \text { if } i \equiv 3(\bmod 4)\end{array}\right.$

and for all $i=1,2,3, \ldots, n-1, \emptyset\left(v_{i} w_{i}\right)=\emptyset\left(v_{i}^{f} w_{i}^{f}\right)=\emptyset\left(u_{i} t_{i}\right)=$ $\emptyset\left(u_{i} t_{i}^{t}\right)=\emptyset\left(v_{i} v_{i}^{\prime}\right)=1, \emptyset\left(u_{i} v_{i}\right)=2$ and $\emptyset\left(u_{i+1} v_{i}^{\prime}\right)=3$.

The corona of a quadrilateral snake $\mathrm{QS}_{6}$ as given in Figure 9.

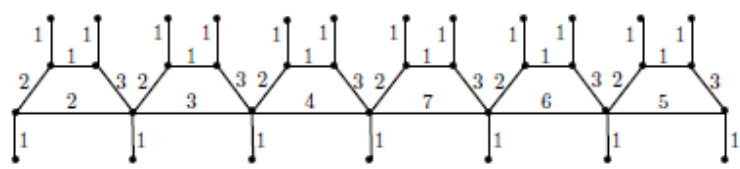

FIGURE 9

Then $S_{\phi}\left(v_{\mathrm{i}}\right)=4, S_{\phi}\left(v_{\mathrm{i}}^{f}\right)=5, S_{\phi}\left(w_{\mathrm{i}}\right)=S_{\phi}\left(w_{\mathrm{i}}^{t}\right)=S_{\phi}\left(t_{\mathrm{i}}\right)$ $=S_{\phi}\left(t_{i}^{t}\right)=1$, for all $i=1,2,3,4, \ldots, n-1, S_{\phi}\left(u_{1}\right)=5, S_{\phi}\left(u_{n}\right)=6$ (or) 8 according as $n \equiv 2(\bmod 6)$ or $n \equiv 1(\bmod 6)$ or $n \equiv 0(\bmod 6)$ where $k \geq 0$ is an integer and for all $i=2,3$, $4, \ldots, n-1$, we have

$S_{\emptyset}\left(u_{i}\right)=\emptyset\left(u_{i} v_{i-1}^{\prime}\right)+\emptyset\left(u_{i} v_{i}\right)+\emptyset\left(u_{i} u_{i+1}\right)+\emptyset\left(u_{i} u_{i-1}\right)+$ $\emptyset\left(u_{i} t_{i-1}^{t}\right)$

$S_{\phi}\left(u_{i}\right)= \begin{cases}9 & \text { if } i \equiv 2(\bmod 4) \\ 11 & \text { if } i \equiv 0(\bmod 4) \\ 13 & \text { otherwise. }\end{cases}$

Clearly, the weights of any two adjacent vertices are coprime and hence $\varnothing$ is a coprime irregular edge-weighting of corona of a quadrilateral snake. So that the corona of a quadrilateral snake is coprime irregular edge-weighting.

\section{CONCLUSION AND SCOPE}

In this paper, we have introduced a coprime irregular edge weighting of graphs and proved some classes of graphs are coprime irregular. Even though, there is a wide scope for further research on this parameter. The following are some interesting directions for future research.

1. Find more classes of coprime irregular graphs.

2. Obtaining a necessary or sufficient condition for a graph to be coprime irregular is worthy trying.

3. It seems to us that the problem of characterizing trees which are coprime irregular would be very interesting.

\section{ACKNOWLEDGMENT}

The author wishes to thank the Management for their motivation and support for this research work.

\section{REFERENCES}

1. G. Chartrand and Lesniak., Graphs and Digraphs, Fourth edition, CRC press, Boca Raton, 2005.

2. J. A. Gallian, A dynamic survey of graph labeling, Electron. J. Combinator., 15 (2008), 190.

\section{AUTHORS PROFILE}

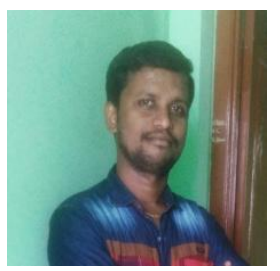

Dr. S. Saravanakumar received his UG, PG and M.Phil Degrees in Mathematics from The Madura College, Madurai in the years 2008, 2010 and 2011 respectively. He pursued his Ph.D. from Madurai Kamaraj University in 2016 and he has published around 6 research articles in reputed $\mathrm{SCI}$ and Scopus indexed journals. His current research areas are coloring, decomposition and domination related parameters in graphs. He is currently working as an Assistant Professor in Mathematics at Kalasalingam

Academy of Research And Education, Krishnankoil- 626126, India. 